\title{
Approximate Confidence Interval for the Reciprocal of a Normal Mean with a Known Coefficient of Variation
}

\author{
Wararit Panichkitkosolkul ${ }^{1}$
}

\begin{abstract}
An approximate confidence interval for the reciprocal of a normal population mean with a known coefficient of variation is proposed. This has applications in the area of nuclear physics, agriculture and economic when the researcher knows the coefficient of variation. The proposed confidence interval is based on the approximate expectation and variance of the estimator by Taylor series expansion. A Monte Carlo simulation study was conducted to compare the performance of the proposed confidence interval with the existing confidence interval. Simulation results show that the proposed confidence interval performs as well as the existing one in terms of coverage probability. However, the approximate confidence interval is very easy to calculate compared with the exact confidence interval.
\end{abstract}

\section{Introduction}

The reciprocal of a normal mean is applied in the area of nuclear physics, agriculture and economic. For example, Lamanna et al. (1981) studied a charged particle momentum, $p=\mu^{-1}$ where $\mu$ is the track curvature of a particle. The reciprocal of a normal mean is defined by $\theta=\mu^{-1}$, where $\mu$ is the population mean. Many researchers studied the reciprocal of a normal mean. For instance, Zaman (1981) discussed the estimators without moments in the case of the reciprocal of a normal mean. The maximum likelihood estimate of the reciprocal of a normal mean with a class of zero-one loss functions was proposed by Zaman (1985). Withers and

\footnotetext{
${ }^{1}$ Department of Mathematics and Statistics, Faculty of Science and Technology, Thammasat University, Thailand; wararit@mathstat.sci.tu.ac.th
} 
Nadarajah (2013) presented the theorem to construct the point estimators for the inverse powers of a normal mean.

Wongkhao et al. (2013) proposed two confidence intervals for the reciprocal of a normal mean with a known coefficient of variation. Their confidence intervals can be applied when the coefficient of variation of a control group is known. One of their confidence intervals is developed based on an exact method in which this confidence interval is constructed from the pivotal statistics $Z$, where $Z$ follows the standard normal distribution. The other confidence interval is constructed based on the generalized confidence interval (Weerahandi, 1993). Simulation results show that the coverage probabilities of the two confidence intervals are not significantly different. However, the confidence interval based on the exact method is shorter than the generalized confidence interval. The exact method uses Taylor series expansion to find the expectation and variance of the estimator of $\theta$ and uses these results for constructing the confidence interval for $\theta$. The lower and upper limits of the confidence interval based on the exact method are difficult to compute since they depend on an infinite summation. Therefore, our main aim in this paper is to propose an approximate confidence interval for the reciprocal of a normal mean with a known coefficient of variation. The computation of the new proposed confidence interval is easier than the exact confidence interval proposed by Wongkhao et al. (2013). In addition, we also compare the estimated coverage probabilities of the new proposed confidence interval and existing confidence interval using a Monte Carlo simulation.

The paper is organized as follows. In Section 2, the theoretical background of the existing confidence interval for $\theta$ is discussed. We provide the theorem for constructing the approximate confidence interval for $\theta$ in Section 3. In Section 4, the performance of the confidence intervals for $\theta$ is investigated through a Monte Carlo simulation study. Conclusions are provided in the final section.

\section{Existing Confidence Interval}

In this section, we review the theorem and corollary proposed by Wongkhao et al. (2013) and use these to construct the exact confidence interval for $\theta$. 
Theorem 1. (Wongkhao et al., 2013) Let $Y_{1}, \ldots, Y_{n}$ be a random sample of size $n$ from a normal distribution with mean $\mu$ and variance $\sigma^{2}$. The estimator of $\theta$ is $\hat{\theta}=\bar{Y}^{-1}$ where $\bar{Y}=n^{-1} \sum_{j=1}^{n} Y_{j}$. The expectation of $\hat{\theta}$ and $\hat{\theta}^{2}$ when a coefficient of variation, $\tau=\frac{\sigma}{\mu}$ is known, are respectively

$$
E(\hat{\theta})=\theta\left[1+\sum_{k=1}^{\infty} \frac{(2 k) !}{2^{k} k !}\left(\frac{\tau^{2}}{n}\right)^{k}\right]
$$

and

$$
E\left(\hat{\theta}^{2}\right)=\theta^{2} \sum_{k=1}^{\infty} \frac{(2 k+1) !}{2^{k} k !}\left(\frac{\tau^{2}}{n}\right)^{k}
$$

Proof of Theorem 1 See Wongkhao et al. (2013)

From (1), $\lim _{n \rightarrow \infty} E(\hat{\theta})=\theta$ and $E\left(\frac{\hat{\theta}}{w}\right)=\theta$, where $w=1+\sum_{k=1}^{\infty} \frac{(2 k) !}{2^{k} k !}\left(\frac{\tau^{2}}{n}\right)^{k}$. Thus, the unbiased estimator of $\theta$ is $\frac{\hat{\theta}}{w}=\frac{1}{w \bar{Y}}$.

Corollary 1. From Theorem 1, $\operatorname{var}(\hat{\theta}) \approx \frac{\theta^{2}}{n} \tau^{2}$.

Proof of Corollary 1 See Wongkhao et al. (2013)

Now we will use the fact that, from the central limit theorem,

$$
Z=\frac{\hat{\theta}-\theta}{\sqrt{\operatorname{var}(\hat{\theta})}} \sim N(0,1) \text {. }
$$

Based on Theorem 1 and Corollary 1, we get

$$
Z=\frac{\frac{\hat{\theta}}{w}-\theta}{\sqrt{\frac{\theta^{2}}{n} \tau^{2}}} \sim N(0,1) .
$$

Therefore, the $100(1-\alpha) \%$ exact confidence interval for $\theta$ based on Equation (2) is

$$
C I_{\text {exact }}=\frac{\hat{\theta}}{w} \pm z_{1-\alpha / 2} \sqrt{\frac{\hat{\theta}^{2}}{n} \tau^{2}}
$$

where $w=1+\sum_{k=1}^{\infty} \frac{(2 k) !}{2^{k} k !}\left(\frac{\tau^{2}}{n}\right)^{k}$ and $z_{1-\alpha / 2}$ is the $100(1-\alpha / 2)$ percentile of the standard normal distribution. 


\section{Proposed Confidence Interval}

To find a simple approximate expression for the expectation of $\hat{\theta}$, we use a Taylor series expansion of $\frac{1}{y}$ around $\mu$ :

$$
\left.\frac{1}{y} \approx \frac{1}{y}\right|_{\mu}+\left.(y-\mu) \frac{\partial}{\partial y}\left(\frac{1}{y}\right)\right|_{\mu}+\left.\frac{1}{2}(y-\mu)^{2} \frac{\partial^{2}}{\partial y^{2}}\left(\frac{1}{y}\right)\right|_{\mu}+O\left(\left(\left(y-\mu_{y}\right) \frac{\partial}{\partial y}\right)^{3}\left(\frac{1}{y}\right)\right) .
$$

Theorem 2. Let $Y_{1}, \ldots, Y_{n}$ be a random sample of size $n$ from a normal distribution with mean $\mu$ and variance $\sigma^{2}$. The estimator of $\theta$ is $\hat{\theta}=\bar{Y}^{-1}$ where $\bar{Y}=n^{-1} \sum_{i=1}^{n} Y_{i}$. The approximate expectation and variance of $\hat{\theta}$ when a coefficient of variation, $\tau=\frac{\sigma}{\mu}$ is known, are respectively

and

$$
\begin{gathered}
E(\hat{\theta}) \approx \frac{1}{\mu}\left(1+\frac{\tau^{2}}{n}\right) \\
\operatorname{var}(\hat{\theta}) \approx \frac{\theta^{2}}{n} \tau^{2} .
\end{gathered}
$$

\section{Proof of Theorem 2.}

Consider random variable $\bar{Y}$ where $\bar{Y}$ has support $(0, \infty)$. Let $\hat{\theta}=\bar{Y}^{-1}$. Find approximations for $E(\hat{\theta})$ and $\operatorname{var}(\hat{\theta})$ using Taylor series expansion of $\hat{\theta}$ around $\mu$ as in Equation (3). The mean of $\hat{\theta}$ can be found by applying the expectation operator to the individual terms (ignoring all terms higher than two),

$$
\begin{aligned}
E(\hat{\theta}) & =E\left(\frac{1}{\bar{Y}}\right) \\
& \left.\approx E\left(\frac{1}{\bar{Y}}\right)\right|_{\mu}+\left.E\left[\frac{\partial}{\partial \bar{Y}}\left(\frac{1}{\bar{Y}}\right)(\bar{Y}-E(\bar{Y}))\right]\right|_{\mu}+\left.\frac{1}{2} E\left[\frac{\partial^{2}}{\partial \bar{Y}^{2}}\left(\frac{1}{\bar{Y}}\right)(\bar{Y}-E(\bar{Y}))^{2}\right]\right|_{\mu}+O\left(n^{-1}\right) \\
& \approx \frac{1}{\mu}+0+\frac{1}{2}\left(\frac{2}{(E(\bar{Y}))^{3}} \operatorname{var}(\bar{Y})\right) \\
& =\frac{1}{\mu}+\frac{\operatorname{var}(\bar{Y})}{\mu^{3}} \\
& =\frac{1}{\mu}\left(1+\frac{\sigma^{2}}{n \mu^{2}}\right) \\
& =\frac{1}{\mu}\left(1+\frac{\tau^{2}}{n}\right)
\end{aligned}
$$


An approximation of the variance of $\hat{\theta}$ is obtained by using the first-order terms of the Taylor series expansion:

$$
\begin{aligned}
\operatorname{var}(\hat{\theta}) & =\operatorname{var}\left(\frac{1}{\bar{Y}}\right) \\
& =E\left[\left(\frac{1}{\bar{Y}}-E\left(\frac{1}{\bar{Y}}\right)\right)^{2}\right] \\
& \approx E\left[\left(\frac{1}{\bar{Y}}-\frac{1}{\mu}\right)^{2}\right] \\
& \left.\approx E\left[\left(\frac{1}{\mu}+\frac{\partial}{\partial \bar{Y}}\left(\frac{1}{\bar{Y}}\right)(\bar{Y}-E(\bar{Y}))-\frac{1}{\mu}\right)^{2}\right]\right|_{\mu} \\
& =\left.\left(\frac{\partial}{\partial \bar{Y}}\left(\frac{1}{\bar{Y}}\right)\right)^{2} \operatorname{var}(\bar{Y})\right|_{\mu} \\
& \approx \frac{\operatorname{var}(\bar{Y})}{\mu^{4}} \\
& =\frac{\sigma^{2}}{n \mu^{4}} \\
& =\frac{\theta^{2}}{n} \tau^{2} .
\end{aligned}
$$

It is clear from Equation (4) that $\hat{\theta}$ is asymptotically unbiased $\left(\lim _{n \rightarrow \infty} E(\hat{\theta})=\theta\right)$ and $E\left(\frac{\hat{\theta}}{v}\right)=\theta$, where $v=1+\frac{\tau^{2}}{n}$. Therefore, the unbiased estimator of $\theta$ is $\frac{\hat{\theta}}{v}=\frac{1}{v \bar{Y}}$. From Equation (5), $\hat{\theta}$ is consistent $\left(\lim _{n \rightarrow \infty} \operatorname{var}(\hat{\theta})=0\right)$.

We then apply the central limit theorem and Theorem 2,

$$
Z=\frac{\frac{\hat{\theta}}{v}-\theta}{\sqrt{\frac{\theta^{2}}{n} \tau^{2}}} \sim N(0,1) .
$$

Therefore, it is easily seen that the $(1-\alpha) 100 \%$ approximate confidence interval for $\theta$ is

$$
C I_{\text {approx }}=\frac{\hat{\theta}}{v} \pm z_{1-\alpha / 2} \sqrt{\frac{\hat{\theta}^{2}}{n} \tau^{2}}
$$


where $v=1+\frac{\tau^{2}}{n}$ and $z_{1-\alpha / 2}$ is the $100(1-\alpha / 2)$ percentile of the standard normal distribution.

\section{Simulation Study}

A Monte Carlo simulation was conducted using the $\mathrm{R}$ statistical software [16] version 3.2.1 to compare the estimated coverage probabilities of the new proposed confidence interval and the exact confidence interval. Source code is available in Appendix. The estimated coverage probability (based on $M$ replicates) are given by $\widehat{1-\alpha}=\#(L \leq \theta \leq U) / M$, where $\#(L \leq \theta \leq U)$ denotes the number of simulation runs for which the true reciprocal of a normal mean $\theta$ lies within the confidence interval. From two previous sections, we found that the lengths of both confidence intervals are equal to $2 z_{1-\alpha / 2} \sqrt{\hat{\theta}^{2} \tau^{2} / n}$ which the expected lengths are not considered in simulation study. The sets of normal data were generated with $\theta=0.1,0.2,0.5$, 1,5 and 10, and the coefficient of variation $\tau=0.05,0.1,0.2,0.33,0.5$ and 0.67 . The sample sizes were set at $n=10,20,30,50,100$ and 500. The number of simulation runs was 10,000 and the nominal confidence level $1-\alpha$ was fixed at 0.95 .

The results are demonstrated in Figure 1 and Tables 1-4. Both confidence intervals have estimated coverage probabilities close to the nominal confidence level for almost situations. However, the estimated coverage probabilities of the exact confidence interval are very poor when the coefficient of variation $\tau$ is close to 1 and small sample sizes. Additionally, the estimated coverage probabilities of the confidence intervals do not increase or decrease according to the values of $\tau$ and $n$. The estimated coverage probabilities of the proposed confidence interval are not significantly different from these of the exact confidence interval in any situation. However, the approximate confidence interval is very easy to calculate compared with the exact confidence interval because the exact confidence interval is based on an infinite summation. 

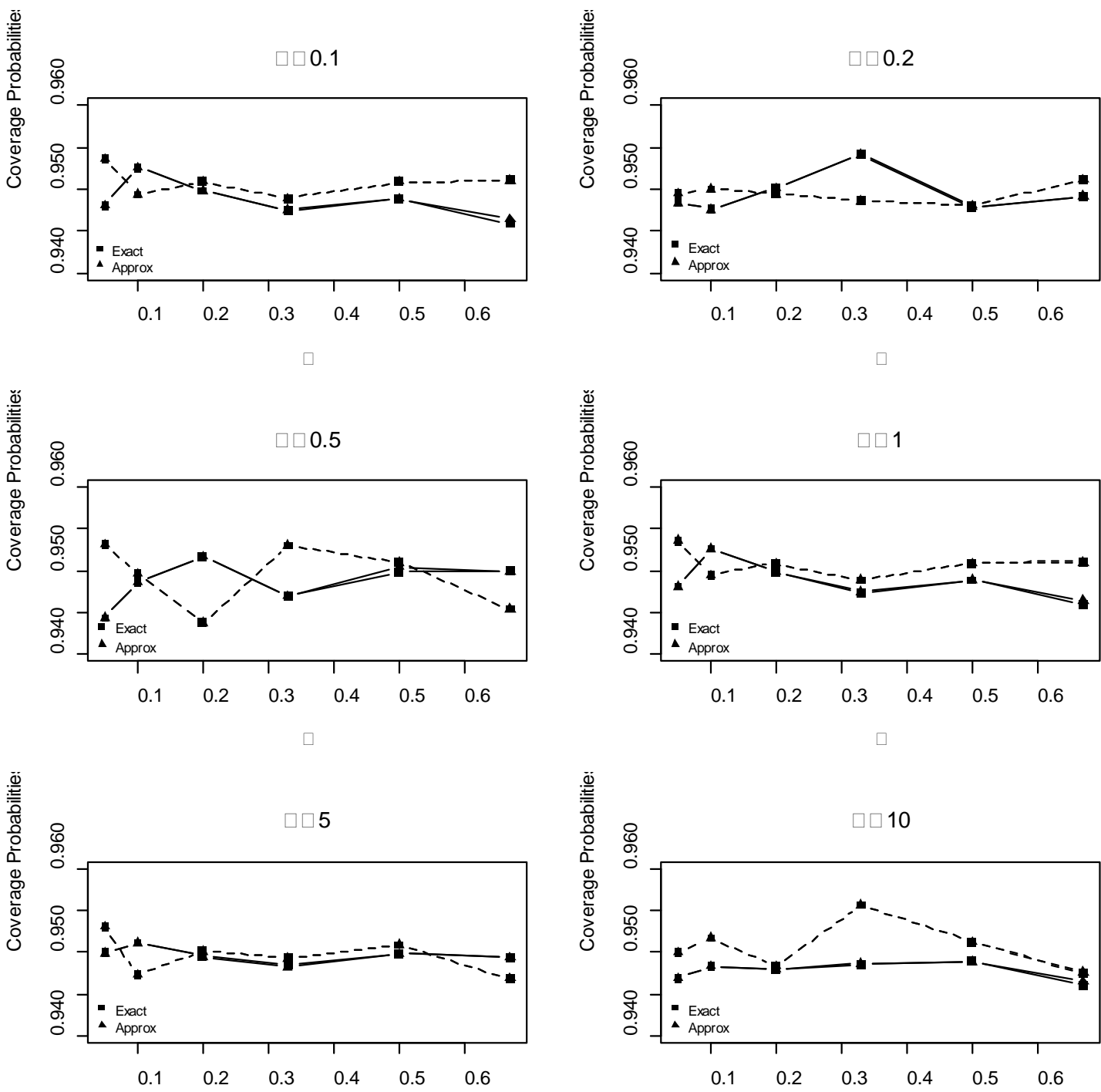

Figure 1: Estimated coverage probabilities of confidence intervals for the reciprocal of a normal mean with a known coefficient of variation when $n=30$ (solid line) and $n=100$ (dash line) 
Table 1: Estimated coverage probabilities of confidence intervals for the reciprocal of a normal mean with a known coefficient of variation when $\theta=0.1$ and 0.2 .

\begin{tabular}{|c|c|c|c|c|c|}
\hline \multirow{2}{*}{$n$} & \multirow[b]{2}{*}{$\tau$} & \multicolumn{2}{|c|}{$\theta=0.1$} & \multicolumn{2}{|c|}{$\theta=0.2$} \\
\hline & & Exact & Approx. & Exact & Approx. \\
\hline \multirow[t]{6}{*}{10} & 0.05 & 0.9475 & 0.9475 & 0.9489 & 0.9489 \\
\hline & 0.10 & 0.9471 & 0.9471 & 0.9493 & 0.9493 \\
\hline & 0.20 & 0.9498 & 0.9499 & 0.9500 & 0.9500 \\
\hline & 0.33 & 0.9482 & 0.9483 & 0.9480 & 0.9486 \\
\hline & 0.50 & 0.9325 & 0.9469 & 0.9334 & 0.9502 \\
\hline & 0.67 & 0.0019 & 0.9456 & 0.0030 & 0.9455 \\
\hline \multirow[t]{6}{*}{20} & 0.05 & 0.9543 & 0.9543 & 0.9489 & 0.9489 \\
\hline & 0.10 & 0.9489 & 0.9489 & 0.9529 & 0.9529 \\
\hline & 0.20 & 0.9519 & 0.9519 & 0.9480 & 0.9479 \\
\hline & 0.33 & 0.9514 & 0.9514 & 0.9492 & 0.9491 \\
\hline & 0.50 & 0.9500 & 0.9505 & 0.9447 & 0.9452 \\
\hline & 0.67 & 0.9475 & 0.9480 & 0.9457 & 0.9459 \\
\hline \multirow[t]{6}{*}{30} & 0.05 & 0.9481 & 0.9481 & 0.9484 & 0.9484 \\
\hline & 0.10 & 0.9526 & 0.9526 & 0.9476 & 0.9476 \\
\hline & 0.20 & 0.9498 & 0.9498 & 0.9501 & 0.9501 \\
\hline & 0.33 & 0.9474 & 0.9475 & 0.9542 & 0.9541 \\
\hline & 0.50 & 0.9489 & 0.9489 & 0.9479 & 0.9479 \\
\hline & 0.67 & 0.9459 & 0.9464 & 0.9490 & 0.9492 \\
\hline \multirow[t]{6}{*}{50} & 0.05 & 0.9474 & 0.9474 & 0.9492 & 0.9492 \\
\hline & 0.10 & 0.9485 & 0.9485 & 0.9500 & 0.9500 \\
\hline & 0.20 & 0.9494 & 0.9494 & 0.9496 & 0.9496 \\
\hline & 0.33 & 0.9499 & 0.9499 & 0.9476 & 0.9475 \\
\hline & 0.50 & 0.9514 & 0.9517 & 0.9496 & 0.9497 \\
\hline & 0.67 & 0.9485 & 0.9486 & 0.9476 & 0.9475 \\
\hline \multirow[t]{6}{*}{100} & 0.05 & 0.9536 & 0.9536 & 0.9495 & 0.9495 \\
\hline & 0.10 & 0.9494 & 0.9494 & 0.9500 & 0.9500 \\
\hline & 0.20 & 0.9509 & 0.9509 & 0.9494 & 0.9494 \\
\hline & 0.33 & 0.9489 & 0.9489 & 0.9486 & 0.9486 \\
\hline & 0.50 & 0.9509 & 0.9509 & 0.9481 & 0.9481 \\
\hline & 0.67 & 0.9511 & 0.9510 & 0.9511 & 0.9511 \\
\hline \multirow[t]{6}{*}{500} & 0.05 & 0.9479 & 0.9479 & 0.9467 & 0.9467 \\
\hline & 0.10 & 0.9488 & 0.9488 & 0.9511 & 0.9511 \\
\hline & 0.20 & 0.9517 & 0.9517 & 0.9511 & 0.9511 \\
\hline & 0.33 & 0.9519 & 0.9519 & 0.9481 & 0.9481 \\
\hline & 0.50 & 0.9469 & 0.9469 & 0.9476 & 0.9476 \\
\hline & 0.67 & 0.9484 & 0.9484 & 0.9480 & 0.9479 \\
\hline
\end{tabular}


Table 2: Estimated coverage probabilities of confidence intervals for the reciprocal of a normal mean with a known coefficient of variation when $\theta=0.5$ and 1 .

\begin{tabular}{|c|c|c|c|c|c|}
\hline \multirow{2}{*}{$n$} & \multirow[b]{2}{*}{$\tau$} & \multicolumn{2}{|c|}{$\theta=0.5$} & \multicolumn{2}{|c|}{$\theta=1$} \\
\hline & & Exact & Approx. & Exact & Approx. \\
\hline \multirow[t]{6}{*}{10} & 0.05 & 0.9489 & 0.9489 & 0.9475 & 0.9475 \\
\hline & 0.10 & 0.9482 & 0.9482 & 0.9471 & 0.9471 \\
\hline & 0.20 & 0.9491 & 0.9491 & 0.9498 & 0.9499 \\
\hline & 0.33 & 0.9462 & 0.9463 & 0.9482 & 0.9483 \\
\hline & 0.50 & 0.9357 & 0.9501 & 0.9325 & 0.9469 \\
\hline & 0.67 & 0.0032 & 0.9471 & 0.0019 & 0.9456 \\
\hline \multirow[t]{6}{*}{20} & 0.05 & 0.9515 & 0.9515 & 0.9543 & 0.9543 \\
\hline & 0.10 & 0.9482 & 0.9481 & 0.9489 & 0.9489 \\
\hline & 0.20 & 0.9502 & 0.9502 & 0.9519 & 0.9519 \\
\hline & 0.33 & 0.9518 & 0.9520 & 0.9514 & 0.9514 \\
\hline & 0.50 & 0.9515 & 0.9518 & 0.9500 & 0.9505 \\
\hline & 0.67 & 0.9445 & 0.9453 & 0.9475 & 0.9480 \\
\hline \multirow[t]{6}{*}{30} & 0.05 & 0.9444 & 0.9444 & 0.9481 & 0.9481 \\
\hline & 0.10 & 0.9486 & 0.9486 & 0.9526 & 0.9526 \\
\hline & 0.20 & 0.9517 & 0.9517 & 0.9498 & 0.9498 \\
\hline & 0.33 & 0.9469 & 0.9470 & 0.9474 & 0.9475 \\
\hline & 0.50 & 0.9499 & 0.9505 & 0.9489 & 0.9489 \\
\hline & 0.67 & 0.9500 & 0.9498 & 0.9459 & 0.9464 \\
\hline \multirow[t]{6}{*}{50} & 0.05 & 0.9474 & 0.9474 & 0.9474 & 0.9474 \\
\hline & 0.10 & 0.9520 & 0.9520 & 0.9485 & 0.9485 \\
\hline & 0.20 & 0.9490 & 0.9490 & 0.9494 & 0.9494 \\
\hline & 0.33 & 0.9485 & 0.9485 & 0.9499 & 0.9499 \\
\hline & 0.50 & 0.9475 & 0.9475 & 0.9514 & 0.9517 \\
\hline & 0.67 & 0.9503 & 0.9502 & 0.9485 & 0.9486 \\
\hline \multirow[t]{6}{*}{100} & 0.05 & 0.9531 & 0.9531 & 0.9536 & 0.9536 \\
\hline & 0.10 & 0.9496 & 0.9496 & 0.9494 & 0.9494 \\
\hline & 0.20 & 0.9438 & 0.9438 & 0.9509 & 0.9509 \\
\hline & 0.33 & 0.9530 & 0.9530 & 0.9489 & 0.9489 \\
\hline & 0.50 & 0.9510 & 0.9510 & 0.9509 & 0.9509 \\
\hline & 0.67 & 0.9454 & 0.9454 & 0.9511 & 0.9510 \\
\hline \multirow[t]{6}{*}{500} & 0.05 & 0.9527 & 0.9527 & 0.9515 & 0.9515 \\
\hline & 0.10 & 0.9469 & 0.9469 & 0.9507 & 0.9507 \\
\hline & 0.20 & 0.9520 & 0.9520 & 0.9442 & 0.9442 \\
\hline & 0.33 & 0.9500 & 0.9500 & 0.9495 & 0.9495 \\
\hline & 0.50 & 0.9507 & 0.9507 & 0.9500 & 0.9500 \\
\hline & 0.67 & 0.9507 & 0.9507 & 0.9519 & 0.9519 \\
\hline
\end{tabular}


Table 3: Estimated coverage probabilities of confidence intervals for the reciprocal of a normal mean with a known coefficient of variation when $\theta=5$ and 10.

\begin{tabular}{|c|c|c|c|c|c|}
\hline \multirow{2}{*}{$n$} & \multirow[b]{2}{*}{$\tau$} & \multicolumn{2}{|c|}{$\theta=5$} & \multicolumn{2}{|c|}{$\theta=10$} \\
\hline & & Exact & Approx. & Exact & Approx. \\
\hline \multirow[t]{6}{*}{10} & 0.05 & 0.9489 & 0.9489 & 0.9508 & 0.9508 \\
\hline & 0.10 & 0.9476 & 0.9476 & 0.9473 & 0.9473 \\
\hline & 0.20 & 0.9516 & 0.9515 & 0.9482 & 0.9482 \\
\hline & 0.33 & 0.9500 & 0.9501 & 0.9497 & 0.9497 \\
\hline & 0.50 & 0.9326 & 0.9475 & 0.9335 & 0.9481 \\
\hline & 0.67 & 0.0020 & 0.9457 & 0.0028 & 0.9505 \\
\hline \multirow[t]{6}{*}{20} & 0.05 & 0.9490 & 0.9490 & 0.9514 & 0.9514 \\
\hline & 0.10 & 0.9490 & 0.9490 & 0.9478 & 0.9478 \\
\hline & 0.20 & 0.9522 & 0.9521 & 0.9440 & 0.9440 \\
\hline & 0.33 & 0.9497 & 0.9497 & 0.9504 & 0.9504 \\
\hline & 0.50 & 0.9474 & 0.9479 & 0.9475 & 0.9479 \\
\hline & 0.67 & 0.9454 & 0.9462 & 0.9472 & 0.9478 \\
\hline \multirow[t]{6}{*}{30} & 0.05 & 0.9499 & 0.9499 & 0.9469 & 0.9469 \\
\hline & 0.10 & 0.9511 & 0.9511 & 0.9483 & 0.9483 \\
\hline & 0.20 & 0.9495 & 0.9495 & 0.9479 & 0.9479 \\
\hline & 0.33 & 0.9485 & 0.9482 & 0.9486 & 0.9487 \\
\hline & 0.50 & 0.9498 & 0.9498 & 0.9489 & 0.9488 \\
\hline & 0.67 & 0.9494 & 0.9494 & 0.9461 & 0.9465 \\
\hline \multirow[t]{6}{*}{50} & 0.05 & 0.9516 & 0.9516 & 0.9512 & 0.9512 \\
\hline & 0.10 & 0.9521 & 0.9521 & 0.9496 & 0.9496 \\
\hline & 0.20 & 0.9510 & 0.9510 & 0.9480 & 0.9480 \\
\hline & 0.33 & 0.9496 & 0.9496 & 0.9481 & 0.9481 \\
\hline & 0.50 & 0.9498 & 0.9497 & 0.9506 & 0.9505 \\
\hline & 0.67 & 0.9513 & 0.9512 & 0.9471 & 0.9471 \\
\hline \multirow[t]{6}{*}{100} & 0.05 & 0.9531 & 0.9531 & 0.9500 & 0.9500 \\
\hline & 0.10 & 0.9473 & 0.9473 & 0.9517 & 0.9517 \\
\hline & 0.20 & 0.9501 & 0.9501 & 0.9483 & 0.9483 \\
\hline & 0.33 & 0.9493 & 0.9493 & 0.9556 & 0.9556 \\
\hline & 0.50 & 0.9509 & 0.9509 & 0.9512 & 0.9512 \\
\hline & 0.67 & 0.9469 & 0.9469 & 0.9475 & 0.9476 \\
\hline \multirow[t]{6}{*}{500} & 0.05 & 0.9497 & 0.9497 & 0.9516 & 0.9516 \\
\hline & 0.10 & 0.9510 & 0.9510 & 0.9505 & 0.9505 \\
\hline & 0.20 & 0.9502 & 0.9502 & 0.9528 & 0.9528 \\
\hline & 0.33 & 0.9486 & 0.9486 & 0.9521 & 0.9521 \\
\hline & 0.50 & 0.9484 & 0.9484 & 0.9525 & 0.9525 \\
\hline & 0.67 & 0.9518 & 0.9518 & 0.9493 & 0.9493 \\
\hline
\end{tabular}

\section{An Illustrative Example}

To illustrate an example of two confidence interval for the reciprocal of a normal mean proposed in the previous section, we used the weights (in kilograms) of 61 one-month old infants listed as follows: 


$$
\begin{array}{llllllllll}
4.610 & 4.550 & 4.460 & 2.940 & 4.160 & 4.110 & 4.410 & 4.800 & 5.130 & 3.670 \\
4.550 & 4.290 & 4.950 & 5.210 & 3.210 & 4.030 & 3.580 & 4.360 & 4.360 & 3.920 \\
4.050 & 4.630 & 3.756 & 4.586 & 5.336 & 2.828 & 4.172 & 4.256 & 4.594 & 4.866 \\
4.784 & 4.520 & 5.238 & 4.320 & 5.330 & 3.836 & 5.916 & 5.010 & 4.344 & 3.496 \\
4.148 & 4.044 & 5.192 & 4.368 & 4.180 & 4.102 & 5.210 & 4.382 & 5.070 & 5.044 \\
3.530 & & & & & & & & &
\end{array}
$$

The data were taken from the study by Ziegler et al. (2007) (cited in Ledolter and Hogg, 2010, p.287). From past experience, we assume that the coefficient of variation of the weights of 61 one-month old infants is about 0.14 . The histogram, density plot, Box-and-Whisker plot and normal quantile-quantile plot are displayed in Figure 2. Algorithm 1 shows the result of the Shapiro-Wilk normality test.

(a)

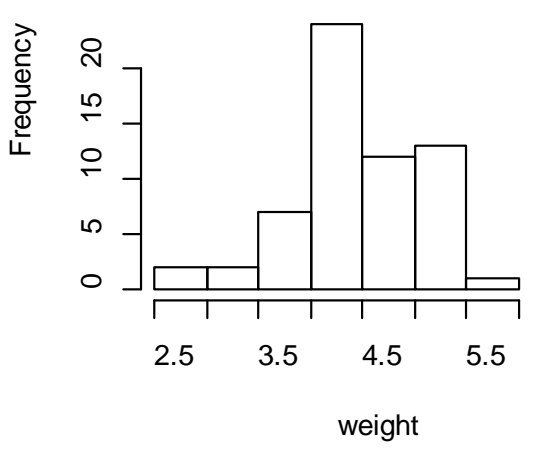

(c)

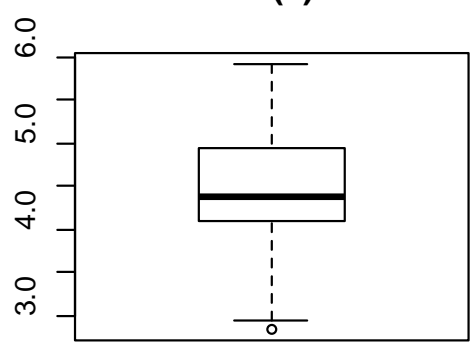

(b)

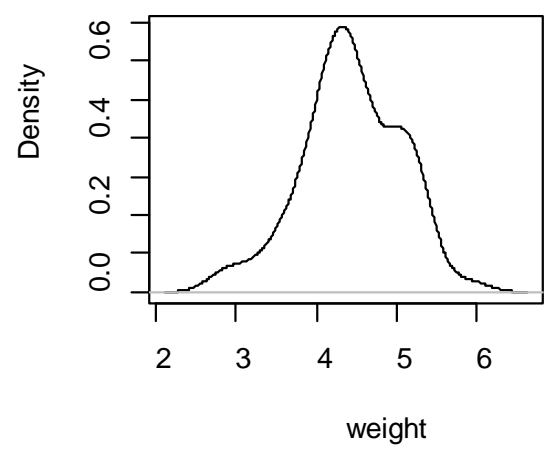

(d)

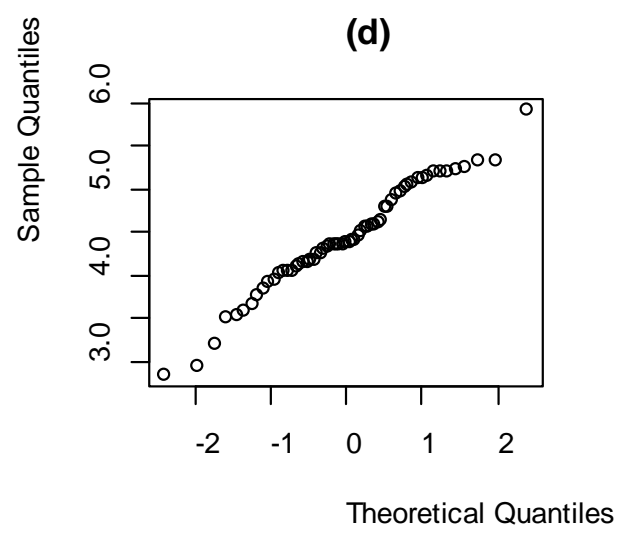

Figure 2: (a) Histogram, (b) density plot, (c) Box-and-Whisker plot and (d) normal quantile-quantile plot of the weight of a one-month old infant 


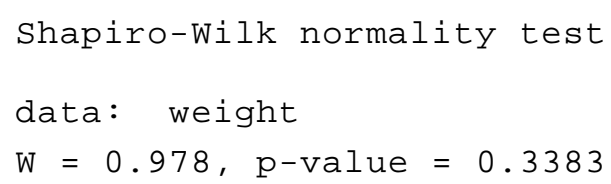

Algorithm 1: Shapiro-Wilk test for normality of the weight of a one-month old infant

The 95\% exact and approximate confidence intervals for the reciprocal of a normal mean are calculated and reported in Table 4. The lower and upper limits of the both confidence intervals are not different.

Table 4: The 95\% confidence intervals for the reciprocal of a normal mean of the weight of a one-month old infant.

\begin{tabular}{|c|cc|c|}
\hline \multirow{2}{*}{ Methods } & \multicolumn{2}{|c|}{ Confidence Intervals } & \multirow{2}{*}{ Lengths } \\
\cline { 2 - 3 } & Lower Limit & Upper Limit & \\
\hline Exact & 0.2176837 & 0.2335416 & 0.0158579 \\
Approximate & 0.2176838 & 0.2335416 & 0.0158578 \\
\hline
\end{tabular}

\section{Conclusions}

In this paper, we proposed an approximate confidence interval for the reciprocal of a normal population mean with a known coefficient of variation. Normally, this arises when the coefficient of variation of the control group is known. The approximate confidence interval proposed uses the approximation of the expectation and variance of the estimator. The proposed new confidence interval is compared with the exact confidence interval constructed by Wongkhao et al. (2013) through a Monte Carlo simulation study. The approximate confidence interval performs as efficiently as the exact confidence interval in terms of coverage probability. Moreover, approximate confidence interval also is easy to compute compared with the exact confidence interval.

\section{Appendix: Source $\mathbf{R}$ code for all confidence intervals}

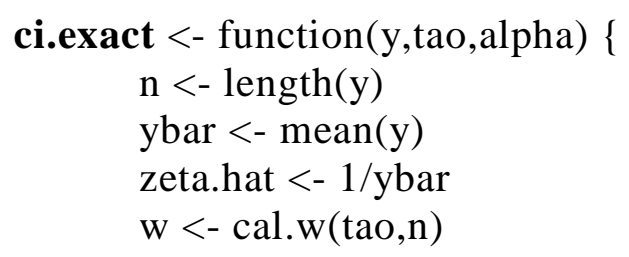




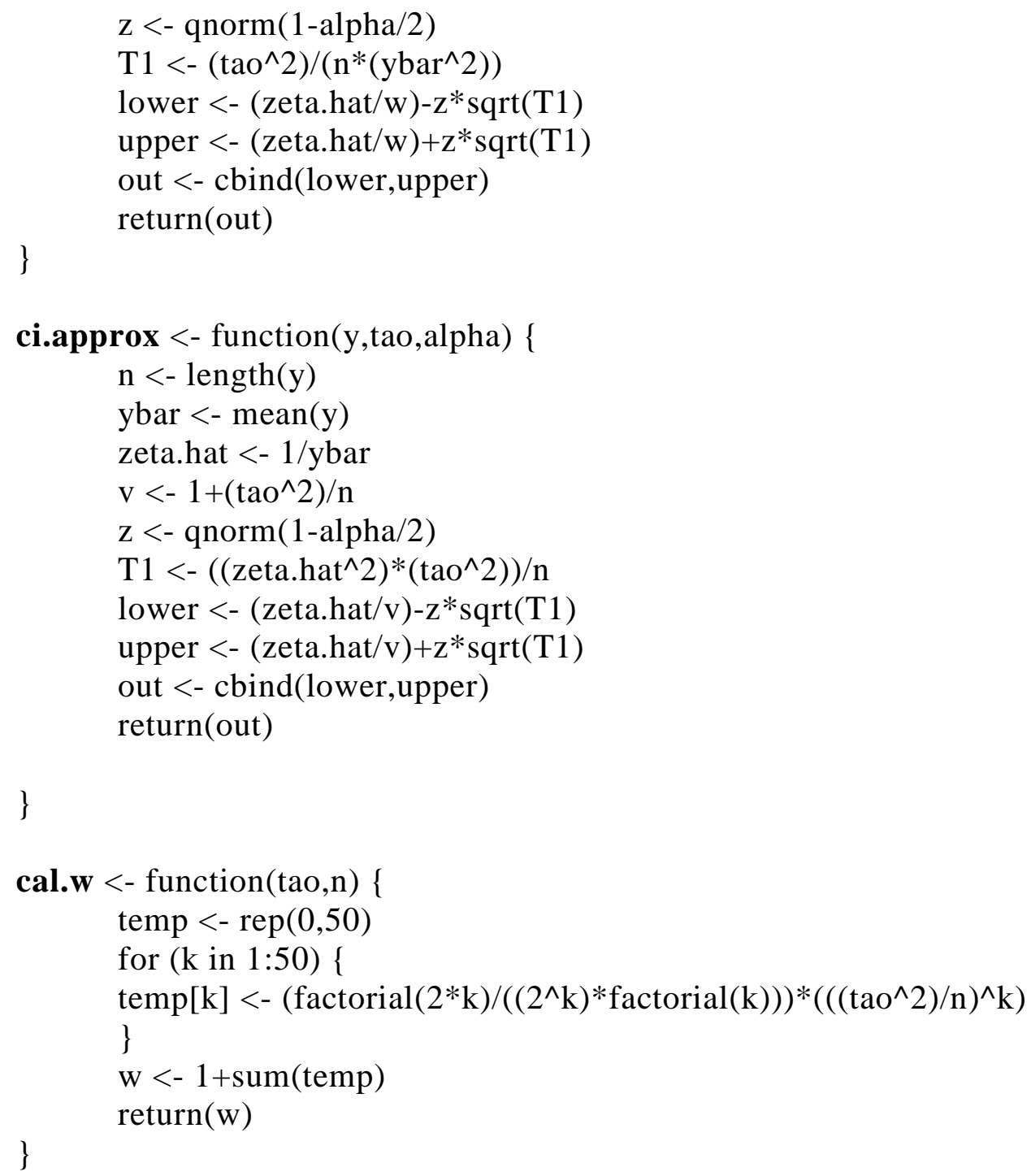

\section{Acknowledgements}

The author is grateful to two anonymous referees for their valuable comments and comments, which have significantly enhanced the quality and presentation of this paper. The author is also thankful for the support in the form of the research funds awarded by Thammasat University.

\section{References}

[1] Ihaka, R. and Gentleman, R. (1996): R: A language for data analysis and graphics. Journal of Computational and Graphical Statistics, 5, 299-314. 
[2] Lamanna, E., Romano, G. and Sgrbi, C. (1981): Curvature measurements in nuclear emulsions. Nuclear Instruments and Methods, 187, 387-391.

[3] Ledolter, L., Hogg, R.V. (2010): Applied Statistics for Engineers and Physical Scientists, Pearson, New Jersey.

[4] Weerahandi, S. (1993): Generalized confidence intervals, Journal of the American Statistical Association, 88, 899-905.

[5] Withers, C.S. and Nadarajah, S. (2013): Estimators for the inverse powers of a normal mean, Journal of Statistical Planning and Inference, 143, 441-455.

[6] Wongkhao, A., Niwitpong, S. and Niwitpong, S. (2013): Confidence interval for the inverse of a normal mean with a known coefficient of variation. International Journal of Mathematical, Computational, Statistical, Natural and Physical Engineer, 7, 877-880.

[7] Zaman, A. (1981): Estimators without moments: the case of the reciprocal of a normal mean. Journal of Econometrics, 15, 289-298.

[8] Zaman, A. (1985): Admissibility of the maximum likelihood estimate of the reciprocal of a normal mean with a class of zero-one loss functions. Sankhya : The Indian Journal of Statistics, Series A, 47, 239-246.

[9] Ziegler, E., Nelson, S.E., Jeter, J.M. (2007): Early iron supplementation of breastfed infants, Department of Pediatrics, University of Iowa, Iowa City, USA. 